\title{
Cardiovascular Modelling and Identification in Septic Shock - Experimental validation
}

17th IFAC World Congress 2008

T. Desaive ${ }^{1}$, B Lambermont ${ }^{1}$, A. Ghuysen ${ }^{1}$, P. Kolh ${ }^{1}$, P. Dauby ${ }^{1}$, C. E. Hann ${ }^{2}$, C.

Starfinger ${ }^{2}$, J. G. Chase ${ }^{2}$, and G. M. Shaw ${ }^{3}$

${ }^{1}$ Hemodynamics Research Laboratory, University of Liège, Belgium ${ }^{2}$ Department of Mechanical Engineering, University of Canterbury, Christchurch, New Zealand ${ }^{3}$ Department of Intensive Care, Christchurch Hospital, Christchurch, New Zealand 


\section{Diagnosis and Treatment}

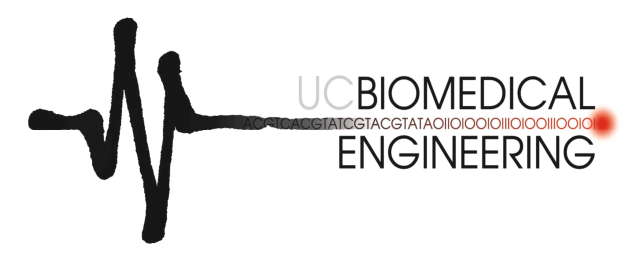

- Problem: Cardiac disturbances difficult to diagnose and treat

- Limited data

- Reflex actions

$\rightarrow$ experience and intuition (mental models)

- Solution: Minimal Model + Patient-Specific Parameter ID

- Interactions of simple models to create complex dynamics

- Primary parameters

- Identification must use common ICU measurements

- E.g. increased resistance in pulmonary artery $\rightarrow$ pulmonary embolism, atherosclerotic heart disease

- However: Identification for diagnosis requires fast parameter ID

- Must occur in "clinical real-time"

- Limits model and method complexity (e.g. parameter numbers, non-linearities, ...)
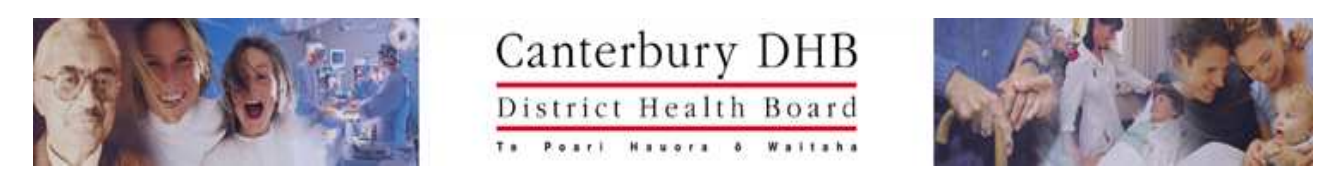


\section{Heart Model}
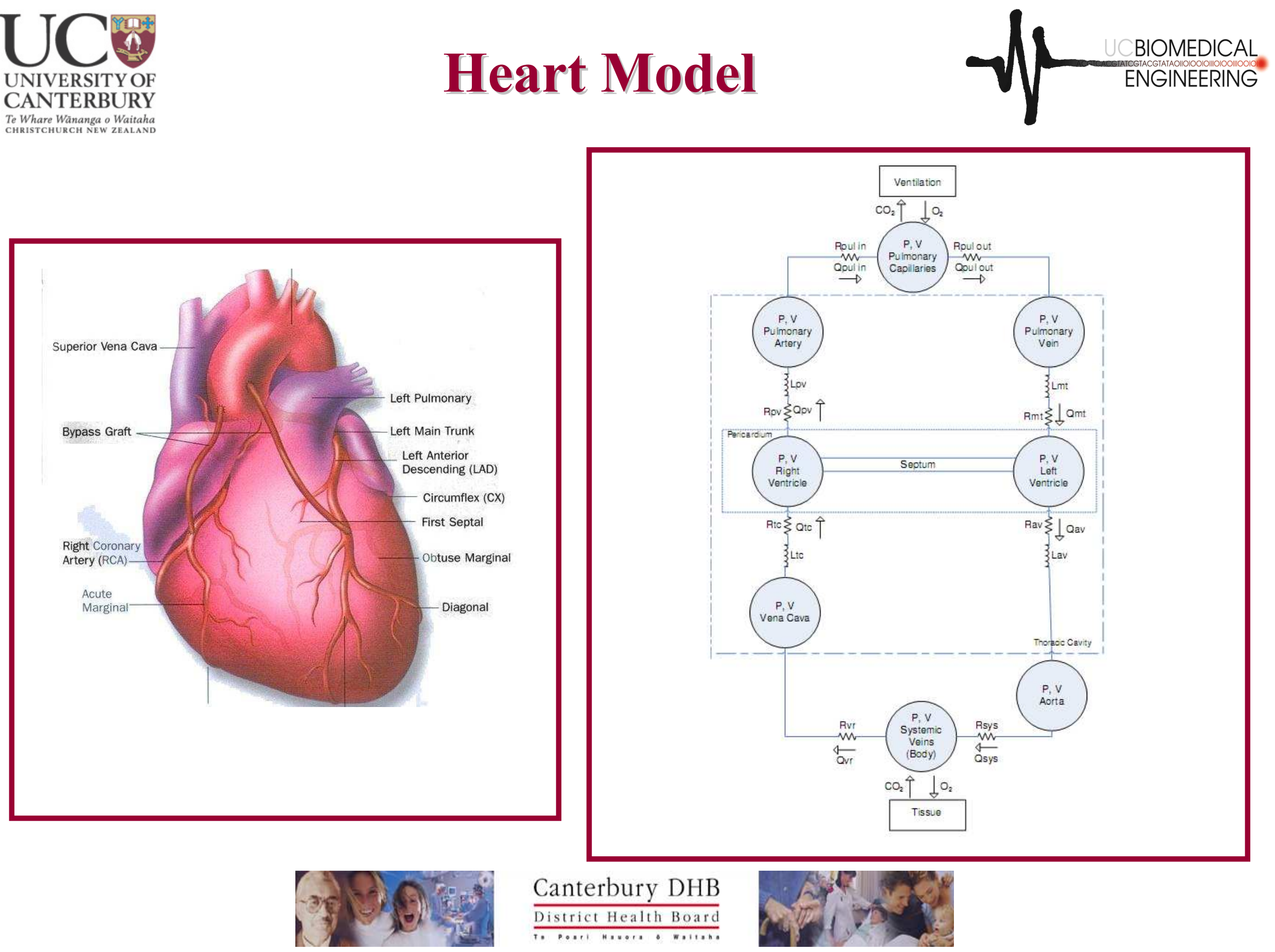

Canterbury DHB $\frac{\text { District Health Board }}{\text { Hown Heor. wotrone }}$

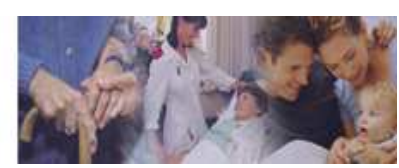




\section{D.E.'s and PV diagram}

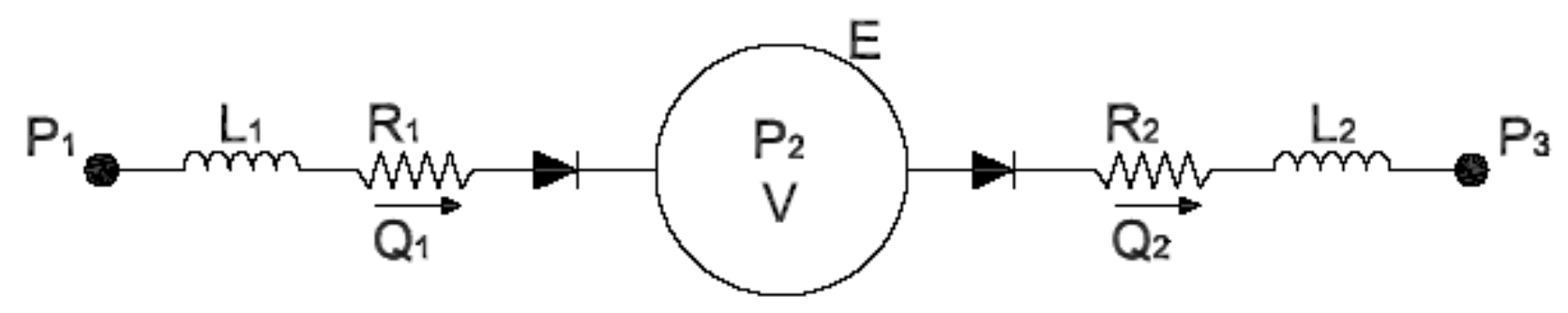

- Open on pressure, close on flow valve law

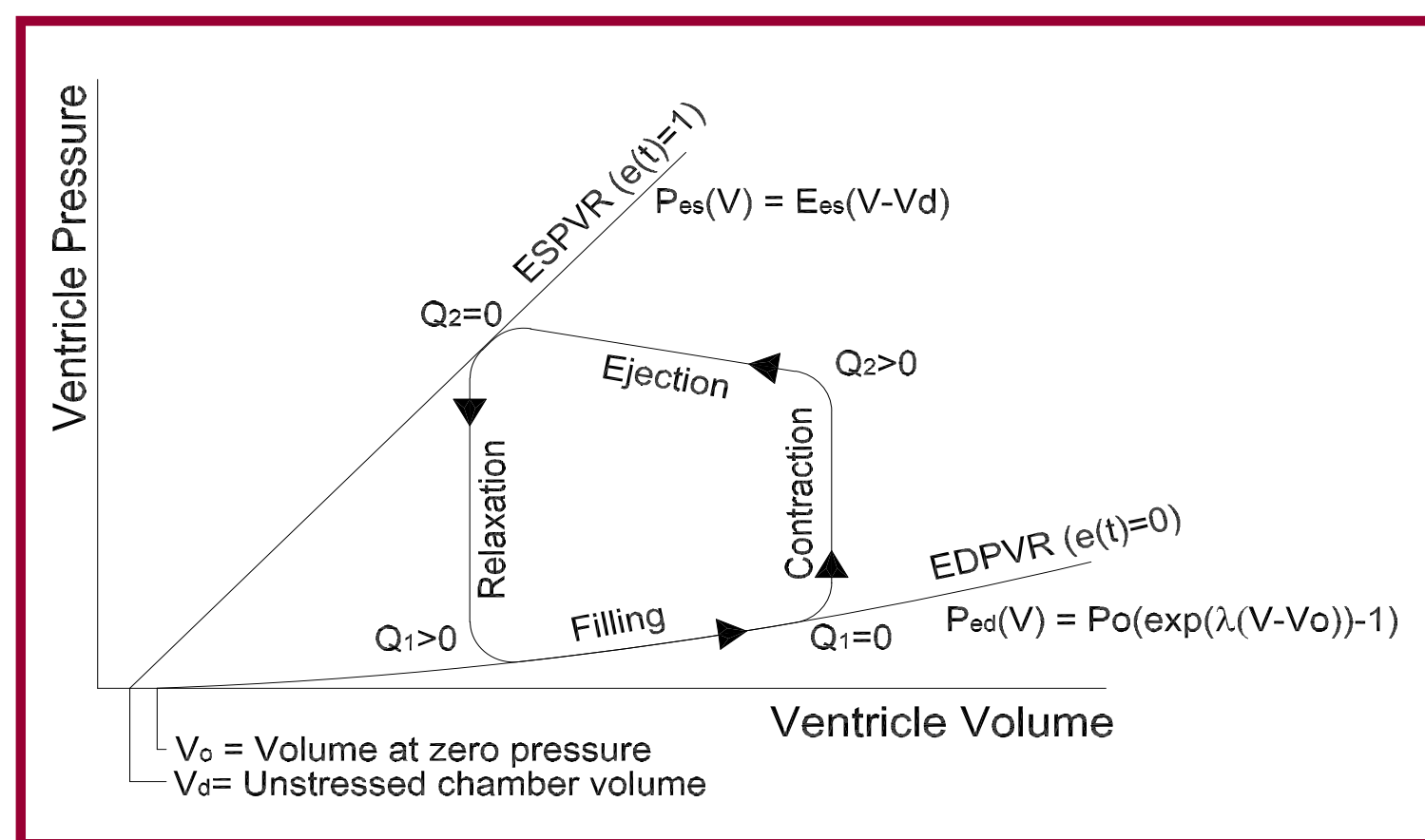

$$
\begin{gathered}
\dot{V}=Q_{1}-Q_{2} \\
\dot{Q}_{1}=\frac{P_{1}-P_{2}-Q_{1} R_{1}}{L_{1}} \\
\dot{Q}_{2}=\frac{P_{2}-P_{3}-Q_{2} R_{2}}{L_{2}} \\
P_{2}=e(t) E_{e s}\left(V-V_{d}\right)+(1-e(t)) P_{0}\left(e^{\lambda\left(V-V_{0}\right)}-1\right), \\
e(t)=e^{-80\left(t-\frac{\text { period }}{2}\right)^{2}}
\end{gathered}
$$

(Cardiac muscle activation) 


\section{Reflex Actions}

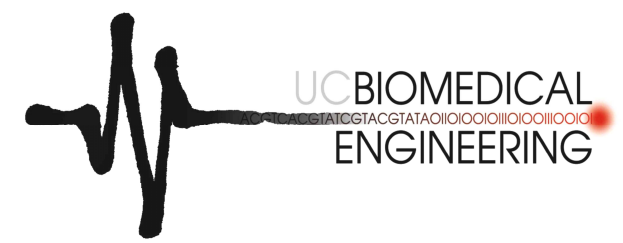

- Vaso-constriction - contract veins

- Venous constriction - increase venous dead space

- Increased HR

- Increased ventricular contractility

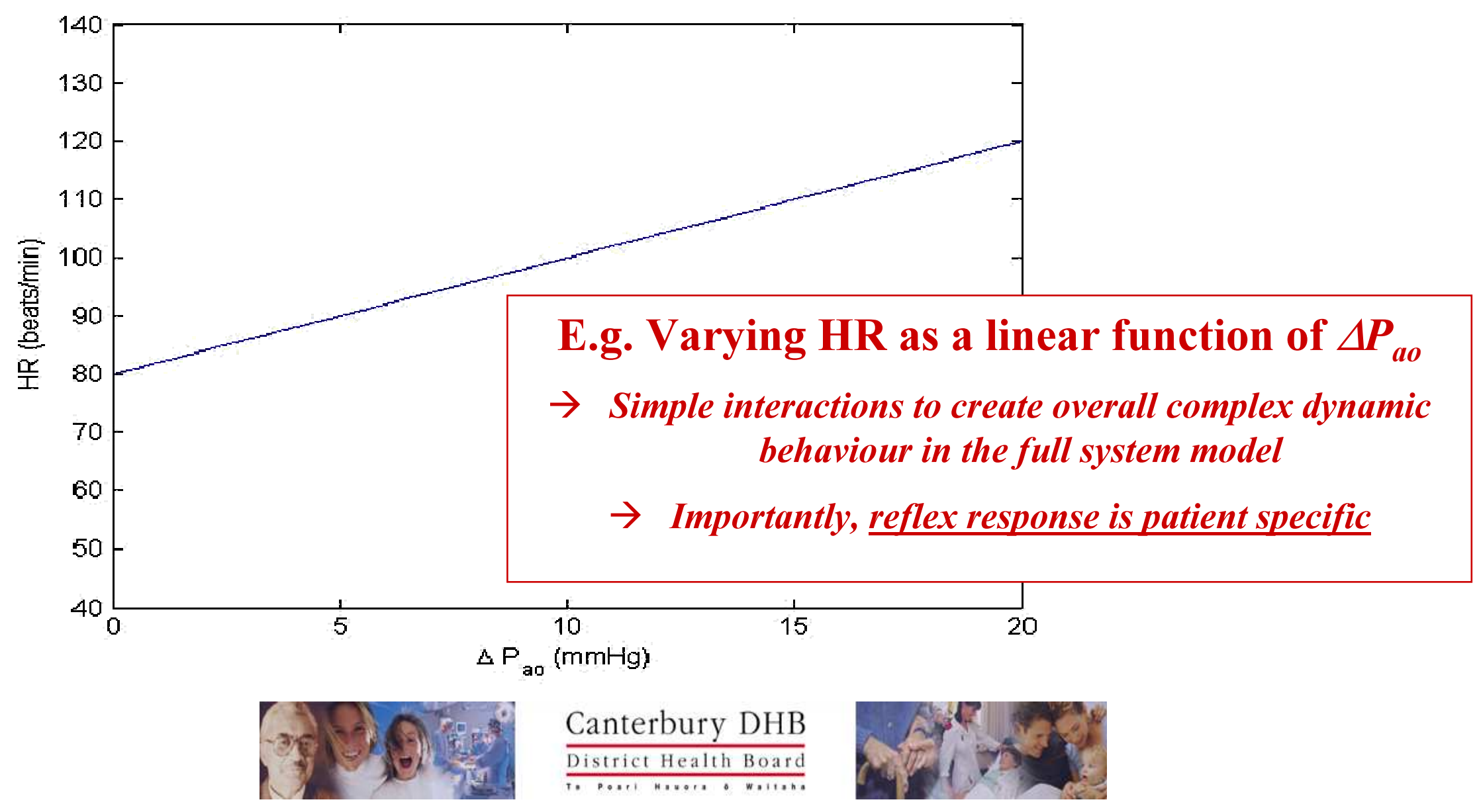




\section{Disease States}

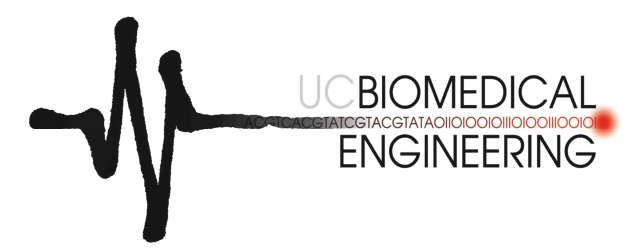

- Pericardial Tamponade:

- Build up of fluid in pericardium

- Decrease: dead space volume V0,pcd

- Pulmonary Embolism:

- Increase: Rpul

\begin{tabular}{ll}
\hline Current Status: \\
\hline- & Clinical results for Pulmonary Embolism \\
- & Clinical results for Septic Shock and \\
& therapy intervention \\
- & Clinical results for PEEP interventions \\
- & Simulated results for others
\end{tabular}

- Cardiogenic shock:

- Not enough oxygen to myocardium (e.g. from blocked coronary artery)

- Decrease: Ees,lvf, Increase: P0,lvf $\rightarrow$ A more complex set of changes/interactions

- Septic shock:

- Blood poisoning

- Decrease: Rsys = systemic resistance

- Hypovolemic shock:

- Severe decrease in total blood volume $=$ sum of individual volumes

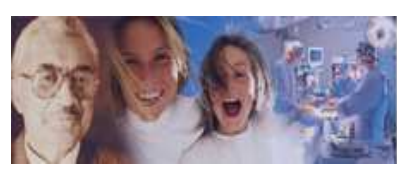




\section{Integral Method - Concept -fft}

- $\dot{x}=a x+b \sin (t)+c, \quad x(0)=1$

$a=-0.5, \quad b=-0.2, \quad c=0.8$

(simple example with analytical solution )

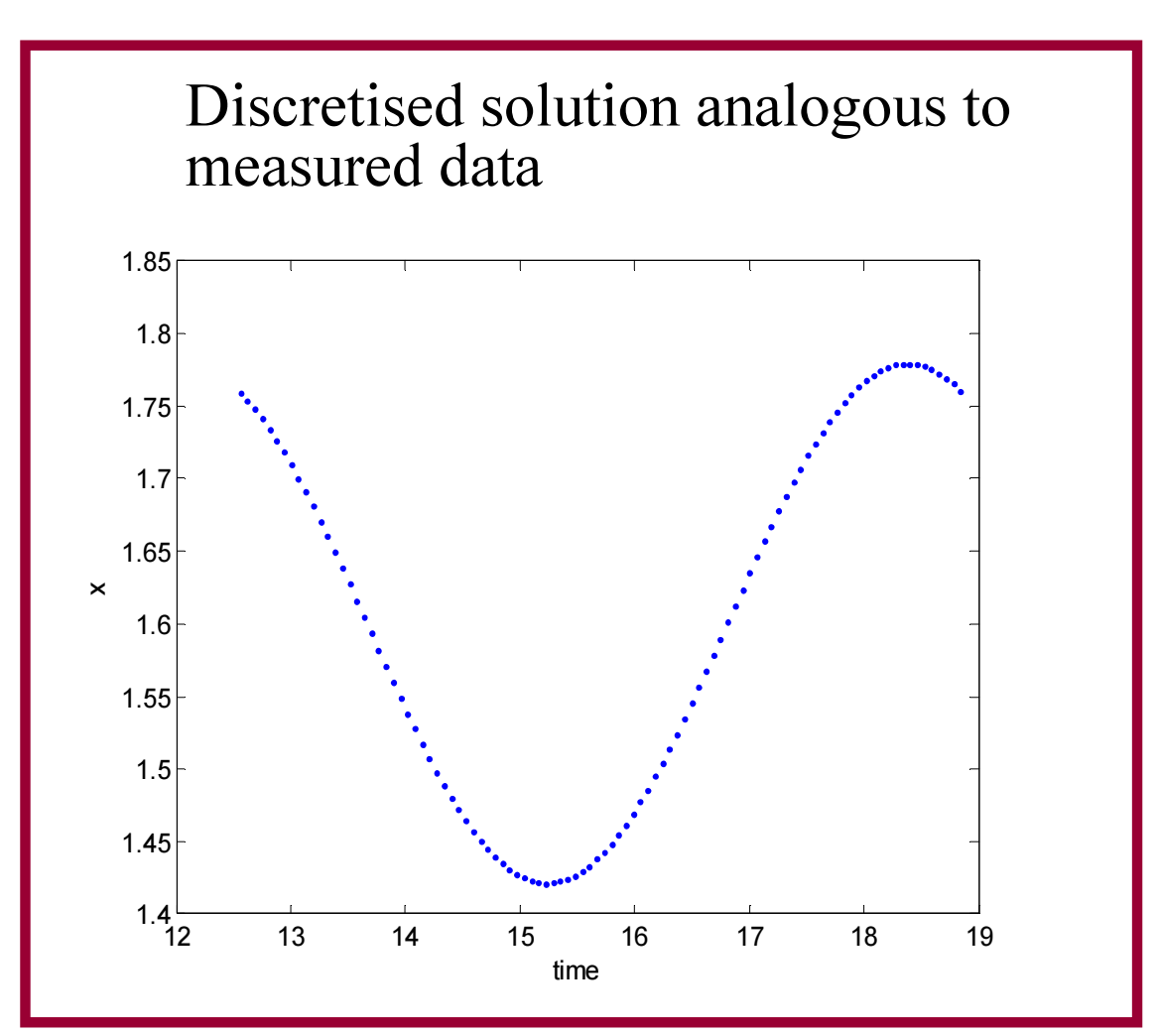

- Work backwards and find a,b,c

- Current method - solve D. E. numerically or analytically

$$
\begin{aligned}
x(t)=\frac{1}{\left(a^{2}+1\right) a}\left(e^{a t}\left(a+c+a b+c a^{2}+a^{3}\right)\right. & \\
& \left.-\left(a b \cos t+b a^{2} \sin t+c a^{2}+c\right)\right)
\end{aligned}
$$

- Find best least squares fit of $x(t)$ to the data

- Non-linear, non-convex optimization, computationally intense

- integral method

- reformulate in terms of integrals

- linear, convex optimization, minimal computation 


\section{Integral Method - Concept - ft}

- Integrate $\dot{x}=a x+b \sin (t)+c$, both sides from $t_{0}$ to $t\left(t_{0}=4 \pi\right)$

$$
\begin{aligned}
& \int_{0}^{1} \dot{x} d t=\int_{0}^{4}(a x+b \sin (t)+c) d t \\
& \Rightarrow x(t)-x\left(t_{0}\right)=a \int_{0}^{t} x d t+b \int_{0}^{t} \sin (t) d t+c \int_{0}^{r} 1 d t \\
& \Rightarrow \quad x(t)=x\left(t_{0}\right)+a \int_{0}^{r} x d t+b\left(\cos \left(t_{0}\right)-\cos (t)\right)+c\left(t-t_{0}\right)
\end{aligned}
$$

- Choose 10 values of $\mathrm{t}$, between $t_{0}=4 \pi$ and $6 \pi$ form 10 equations in 3 unknowns a,b,c

$$
a \int_{t_{0}}^{t} x d t+b\left(1-\cos \left(t_{i}\right)\right)+c\left(t_{i}-t_{0}\right)=x\left(t_{i}\right)-x\left(t_{0}\right), \quad i=1, \ldots, 10
$$




\section{Integral Method - Concept - ft}

$$
\left(\begin{array}{ccc}
\int_{t_{0}}^{t_{1}} x d t & \cos \left(t_{0}\right)-\cos \left(t_{1}\right) & t_{1}-t_{0} \\
\vdots & \vdots & \vdots \\
\int_{t_{0}}^{t_{10}} x d t & \cos \left(t_{0}\right)-\cos \left(t_{10}\right) & t_{10}-t_{0}
\end{array}\right)\left(\begin{array}{l}
a \\
b \\
c
\end{array}\right)=\left(\begin{array}{c}
x\left(t_{1}\right)-x\left(t_{0}\right) \\
\vdots \\
x\left(t_{10}\right)-x\left(t_{0}\right)
\end{array}\right)
$$

- Linear least squares (unique solution)

\begin{tabular}{|c|c|c|l|}
\hline Method & Starting point & CPU time (seconds) & Solution \\
\hline Integral & - & 0.003 & {$[-0.5002,-0.2000,0.8003]$} \\
\hline Non-linear & {$[-1,1,1]$} & 4.6 & {$[-0.52,-0.20,0.83]$} \\
\hline Non-linear & {$[1,1,1]$} & 20.8 & {$[0.75,0.32,-0.91]$} \\
\hline
\end{tabular}

- Integral method is at least 1000-10,000 times faster depending on starting point

- Thus very suitable for clinical application
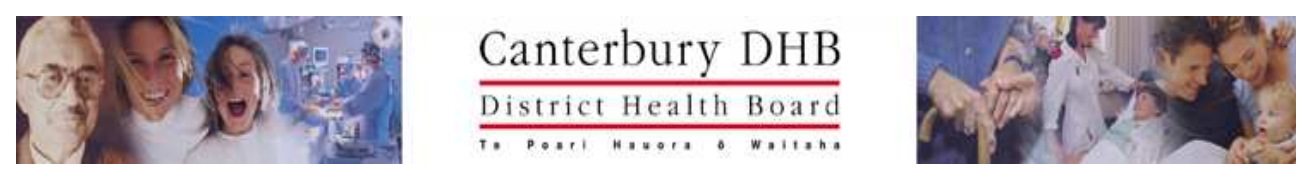


\section{Integral method with discrete data

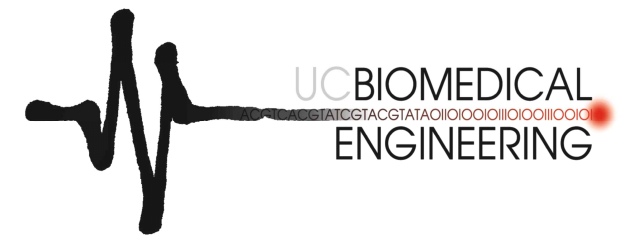

- If only given max and min, scale pre-computed waveforms (cts input)

- Requires typically several iterations - but fast convergence since integrals filter modelling error and noise

- "Simulation" can be replaced by closed form analytical approximations - immediate evaluation at steady state

- $10^{5}-10^{6}$ times faster than standard methods (non-linear regression)

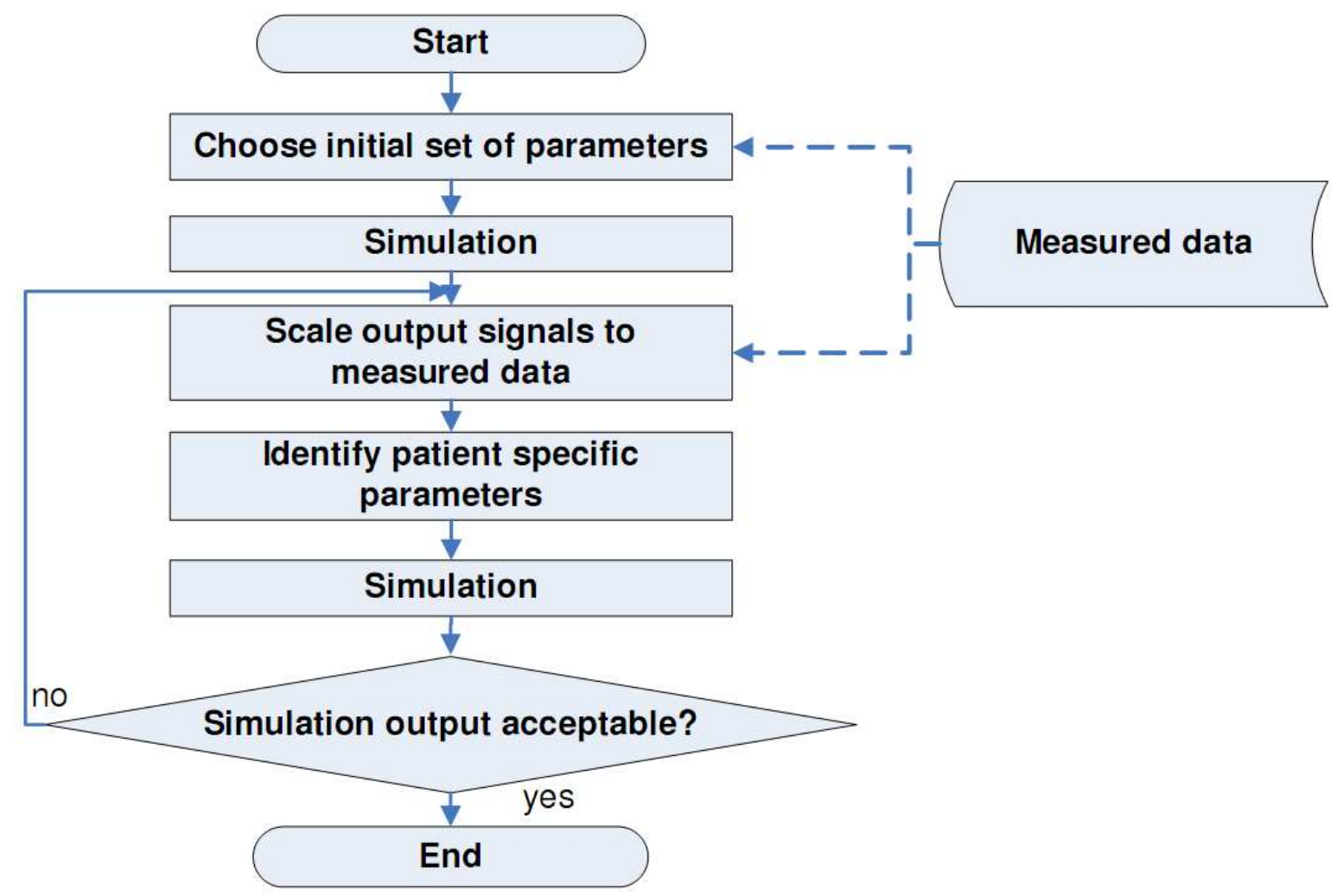


- Septic shock induced in pigs (collaborators in Liege, Belgium)

Open chest

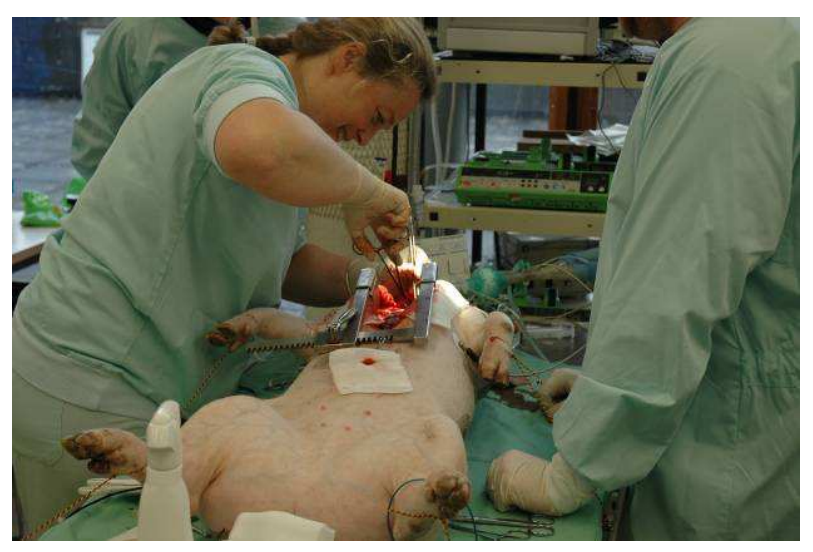

12 Electrode Conductance Catheter (Vlv, Vrv,Plv,Prv)
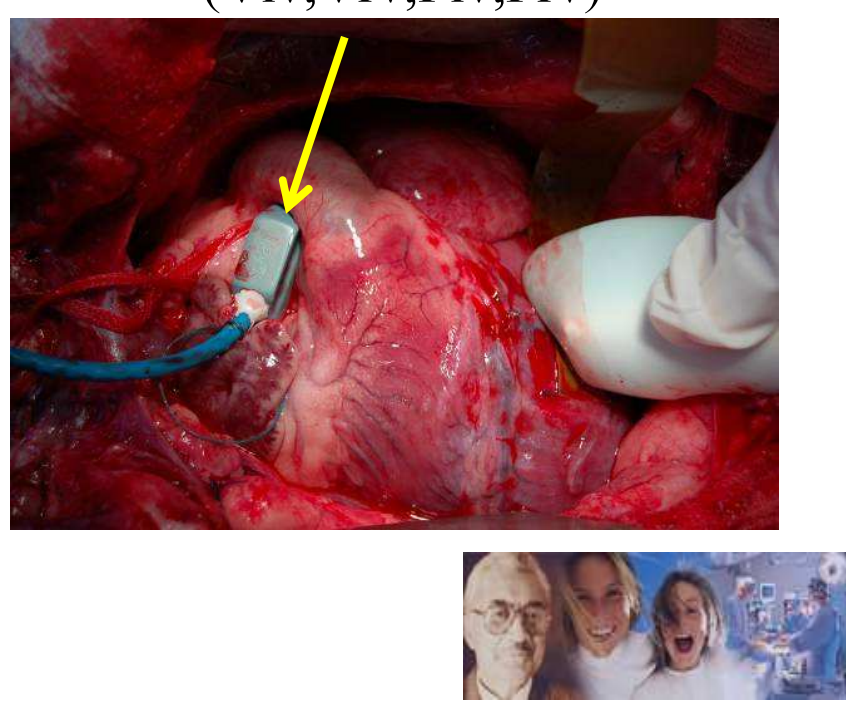

Ventilated and sedated
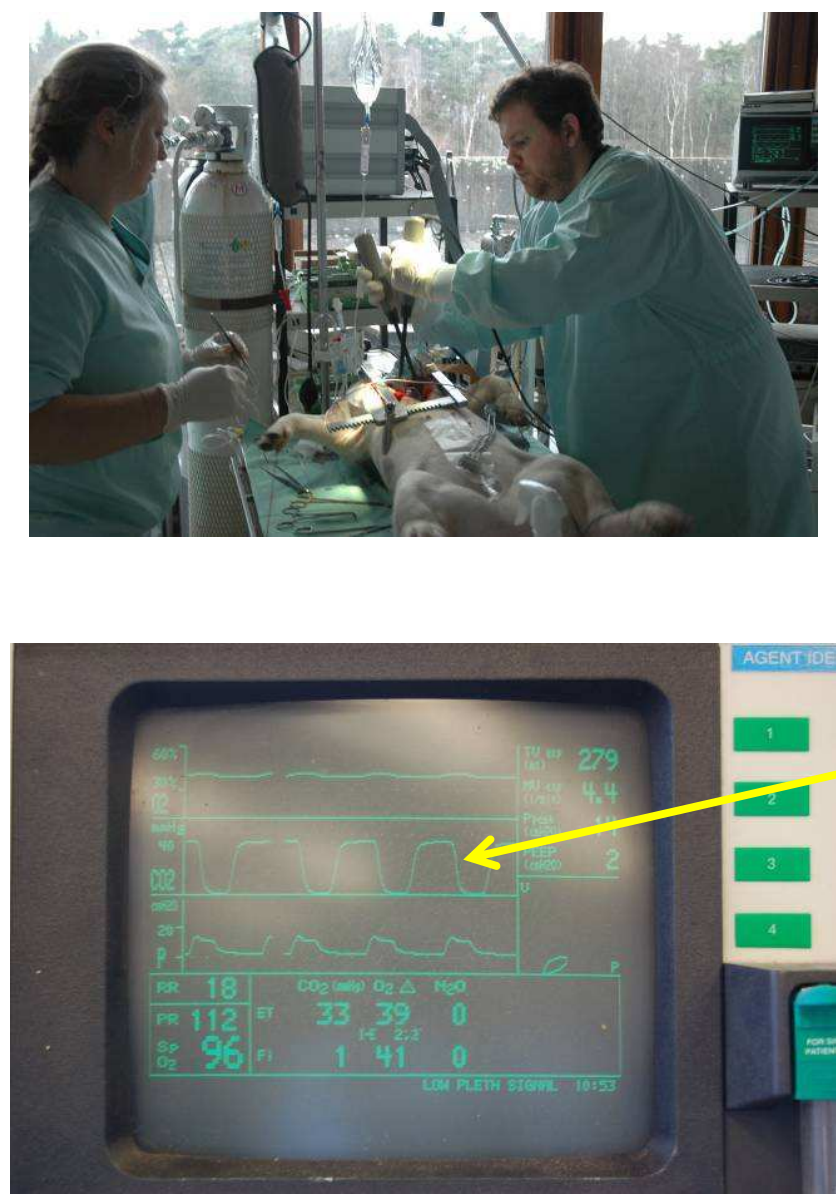

Canterbury DHB $\frac{\text { District Health Board }}{\text { Pote naure wowteno }}$
Also measure

Pao,Ppa 

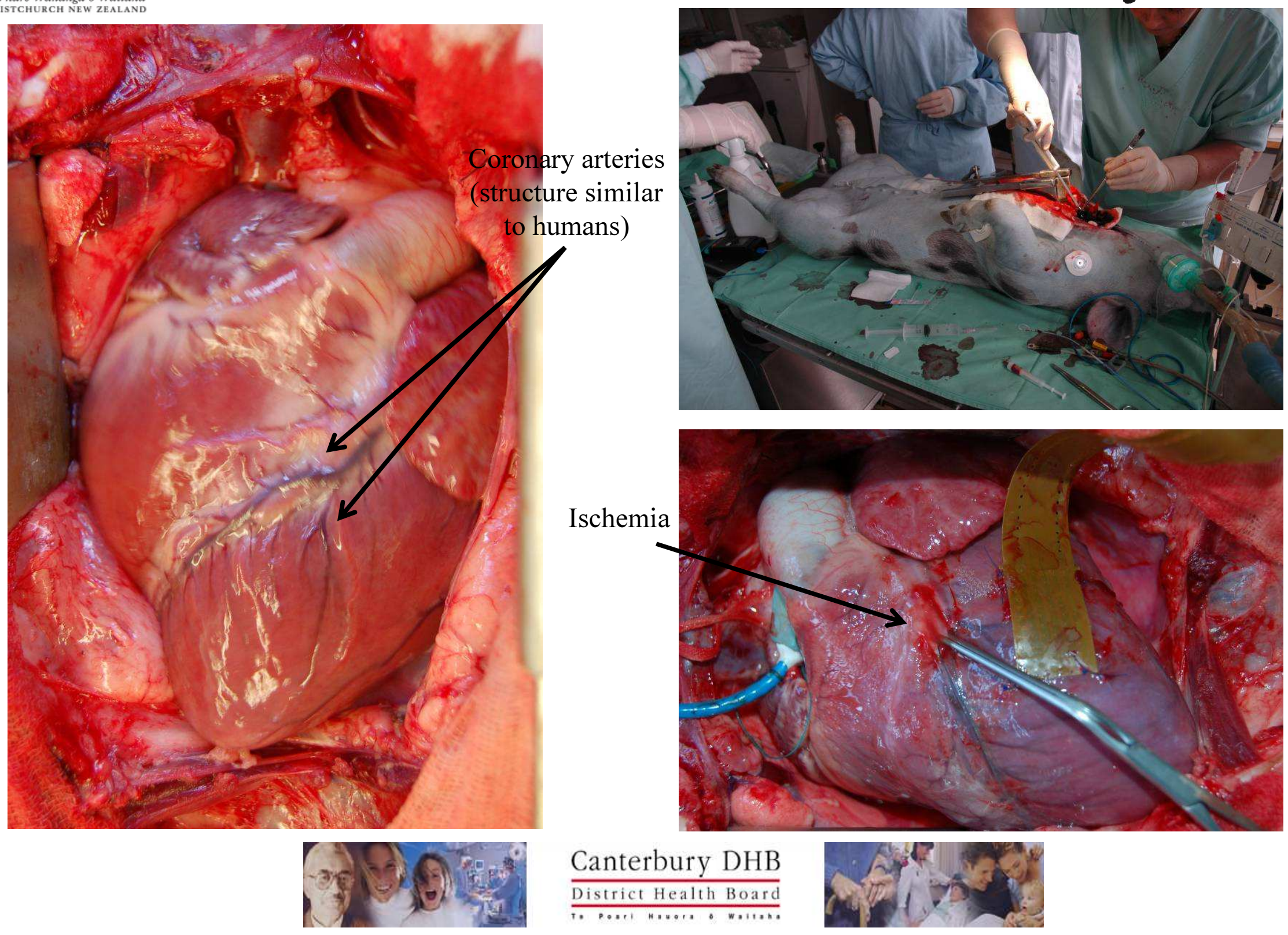


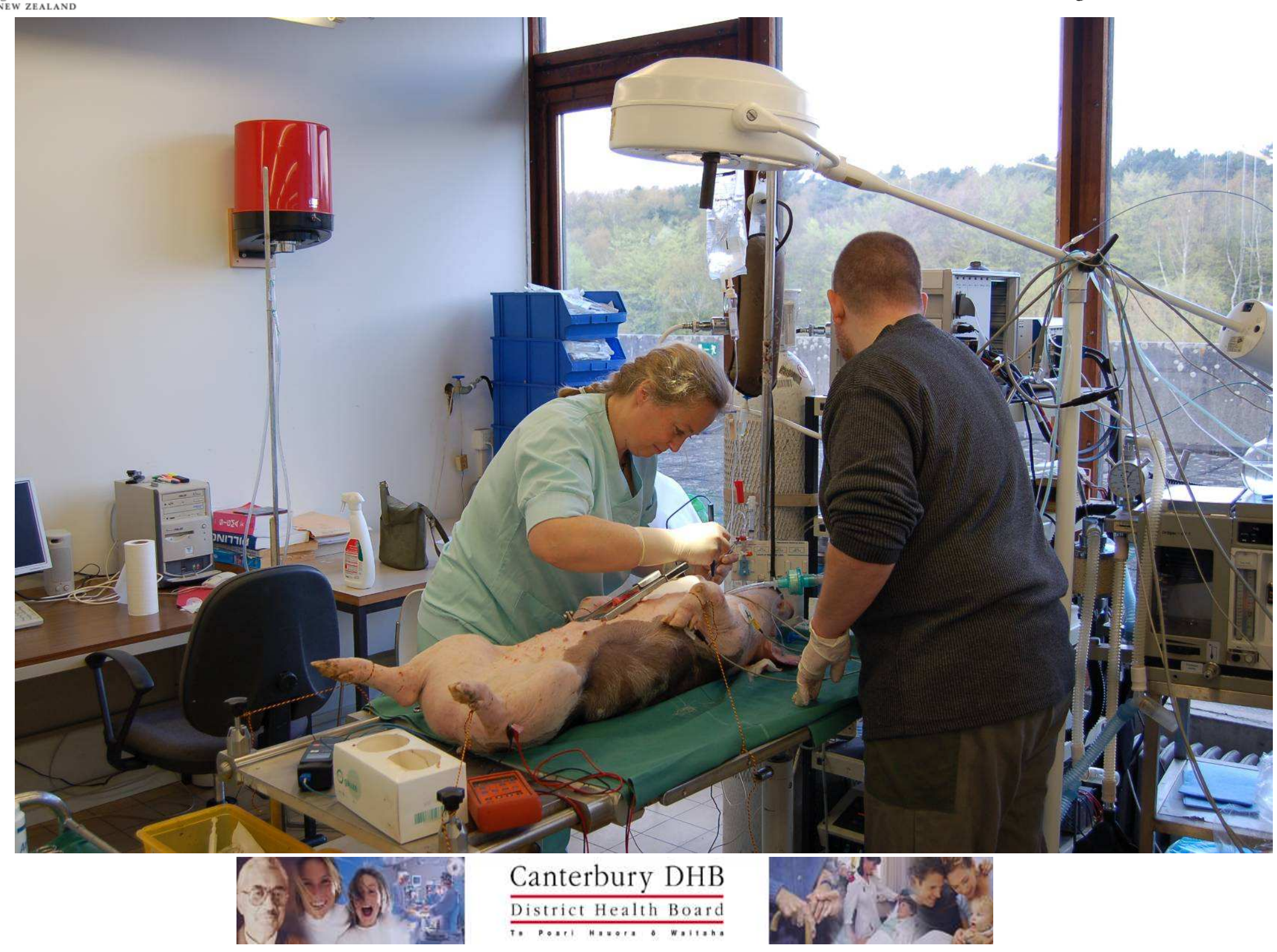




\section{Clinical Results}

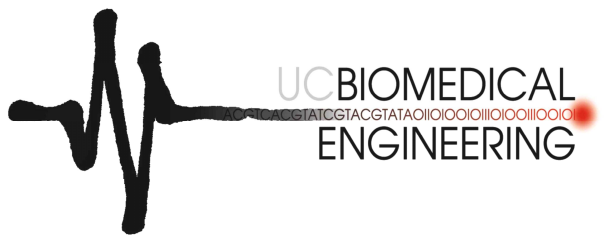

- Use only: Pao, Ppa, $\min / \max (V l v$, Vrv) to ID all parameters

- Measurements available in an ICU

LV volume (0 mins)

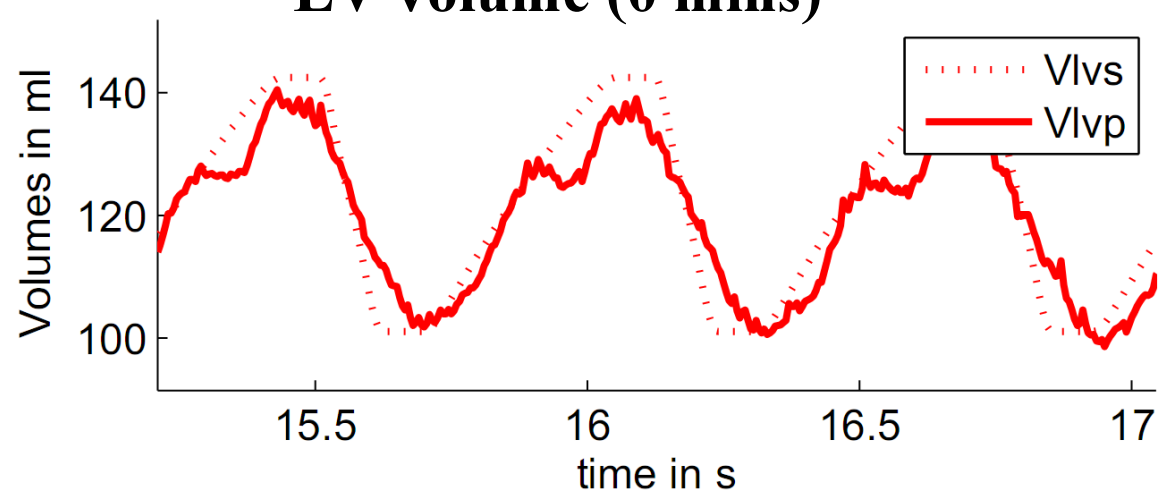

RV pressures (0 mins)

(Plv and Prv are not in ID)

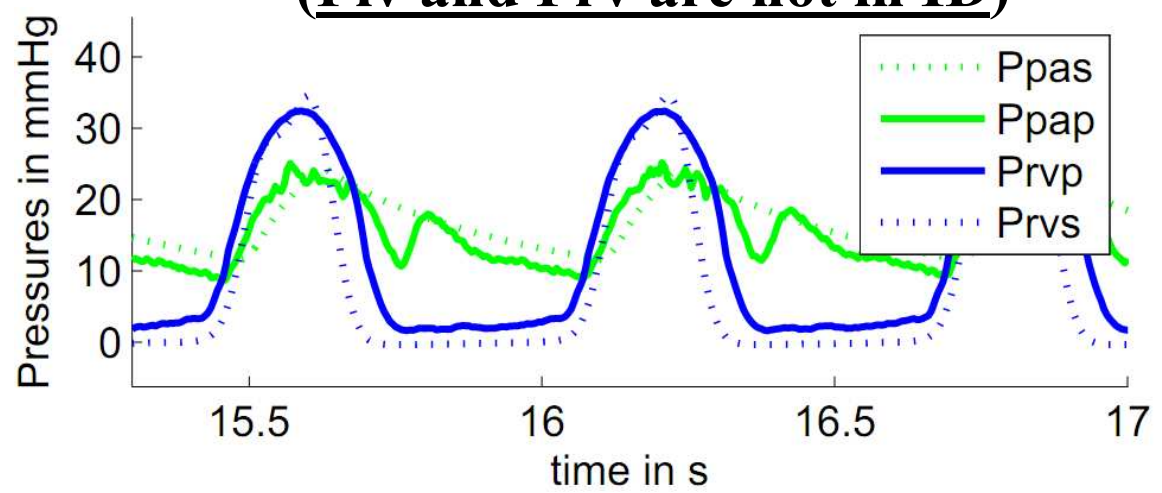

LV volume (120 mins)

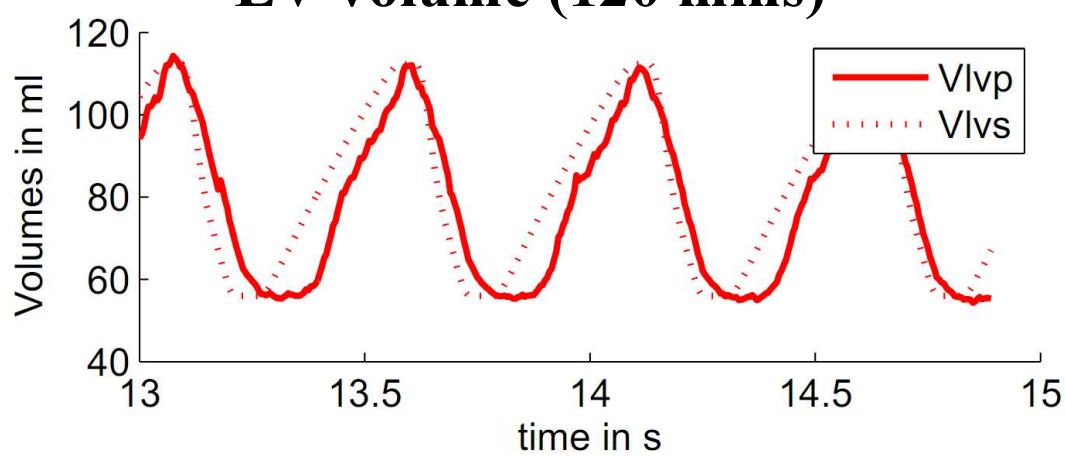

RV pressures (120 mins)

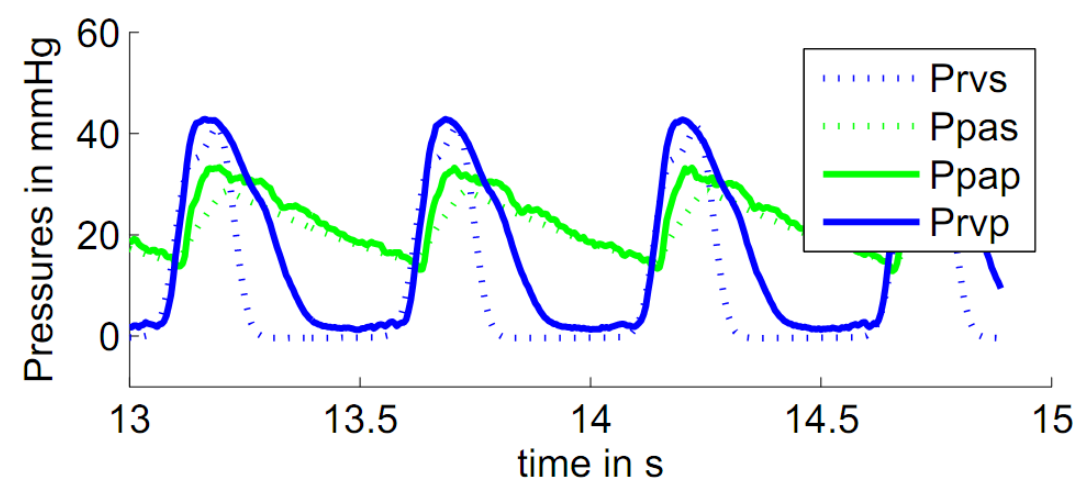

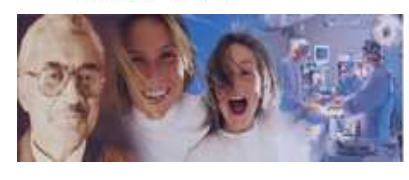

Canterbury DHB District Health Board

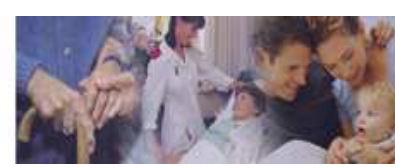



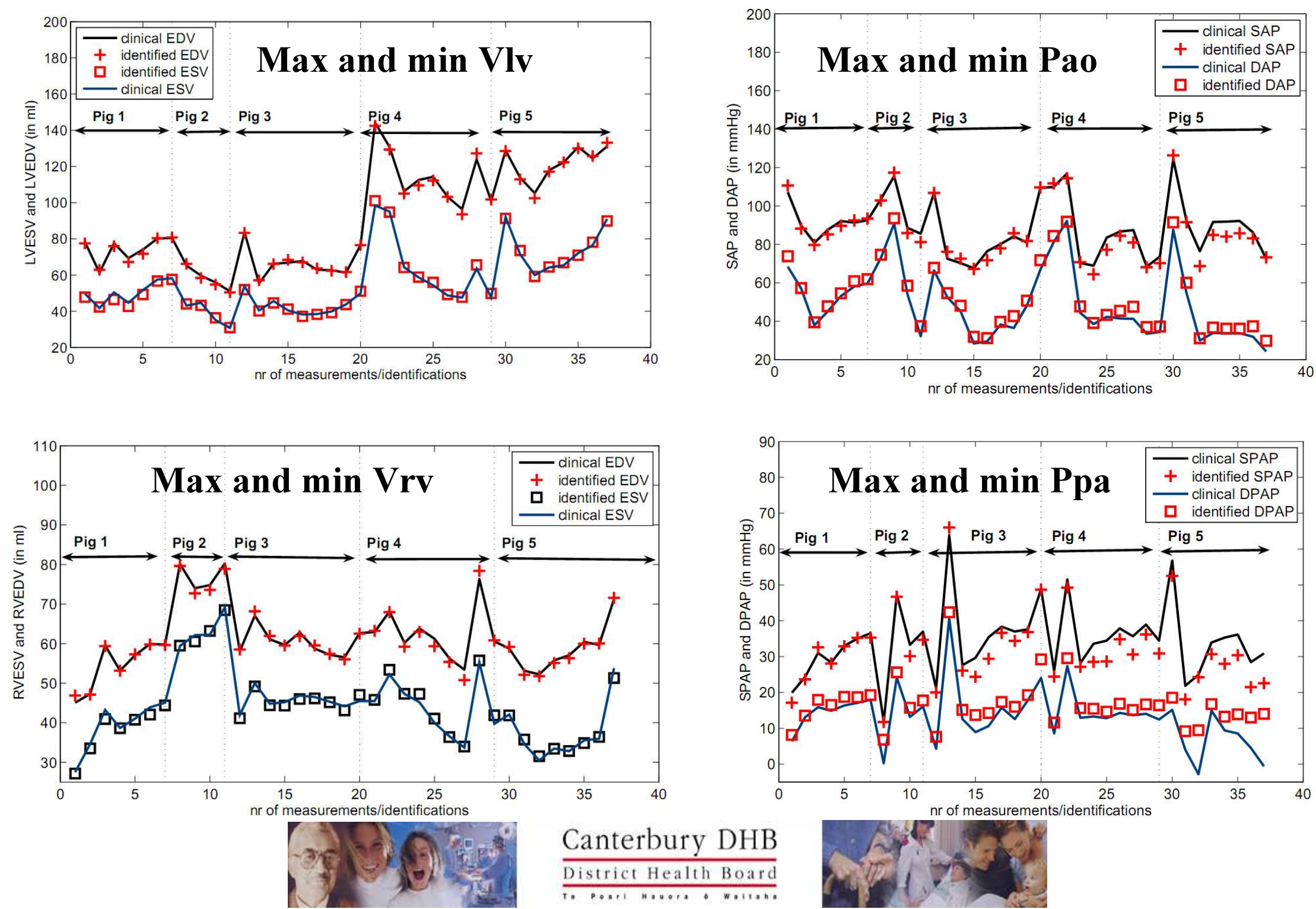


\section{RV-vascular coupling}

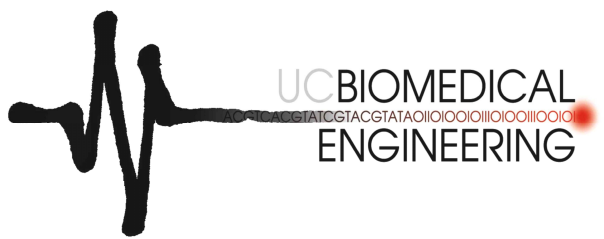

Coupling $=\frac{\text { Contractil ity }}{\text { Afterload }}$

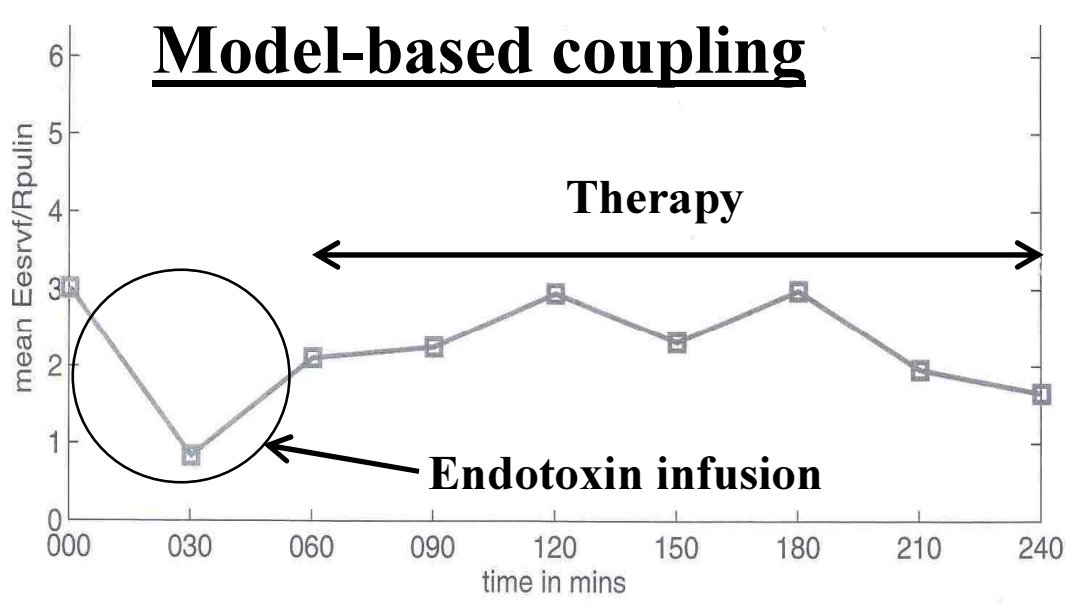

\section{Measured coupling}

Therapy

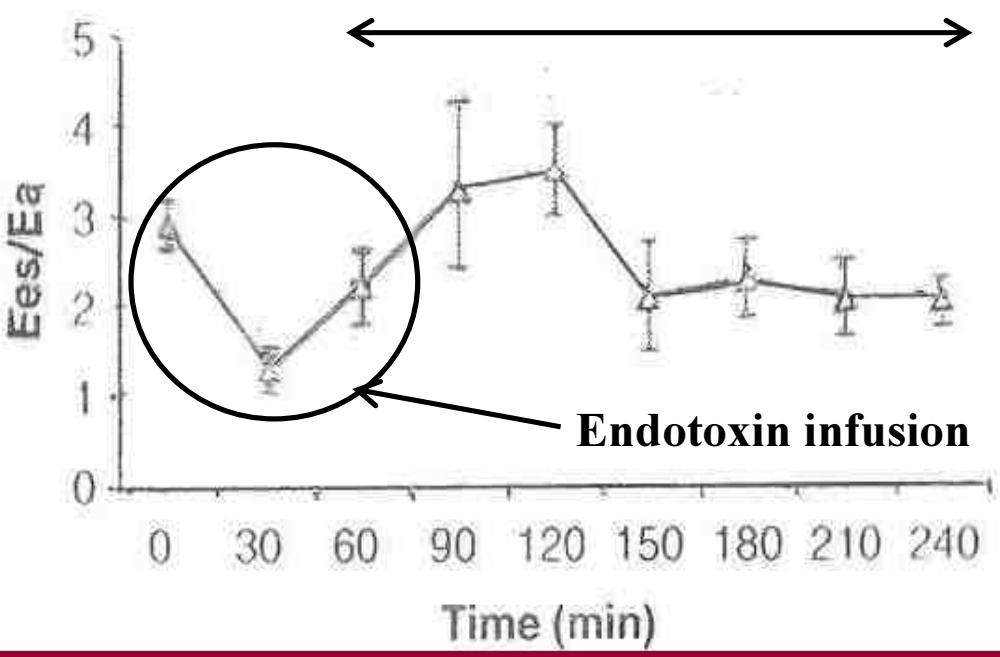

- Five pigs - Endotoxin infusion over first 30 minutes

- Therapy $\rightarrow$ large-pore membrane Hemofiltration from 60-240 minutes

- Model accurately matches all hemodynamic response $<8 \%$ error

- Preserved coupling validated with invasive rapid vena cava occlusion maneuver
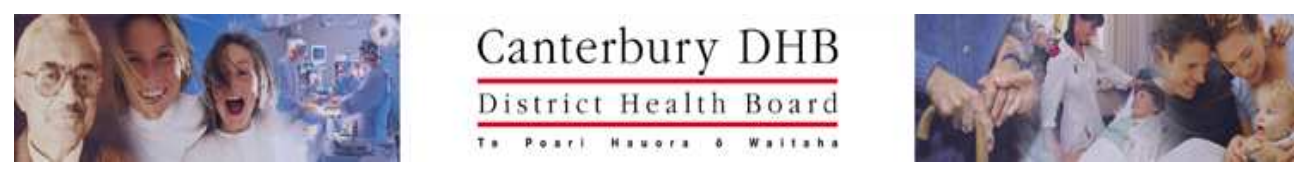


\section{New results for IFAC}

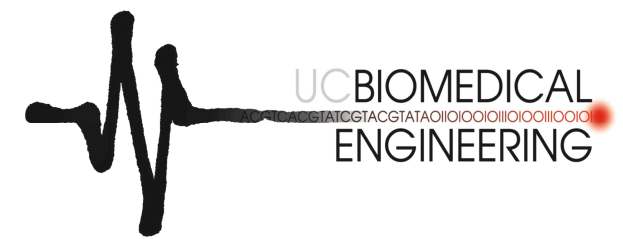

- Using extra measurement of ECG $\rightarrow$ systolic, diastolic timings

- Experimentally derived, driver function shape (Plv/Vlv)
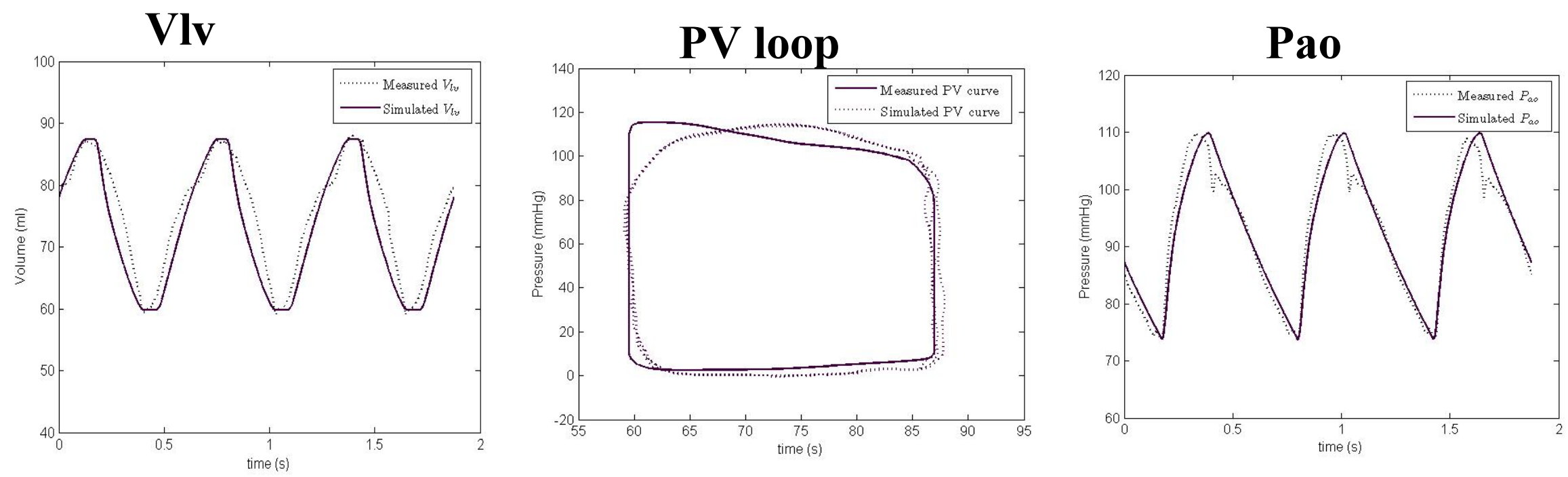

- Don't need max and min Vlv and Vrv. Replace by SV's (much easier to measure!)

- $<10 \%$ change in fitted parameters (including volumes), improved ID of valvular resistances (trade off a little with Ees, significantly with preload parameters, e.g. Ppu)
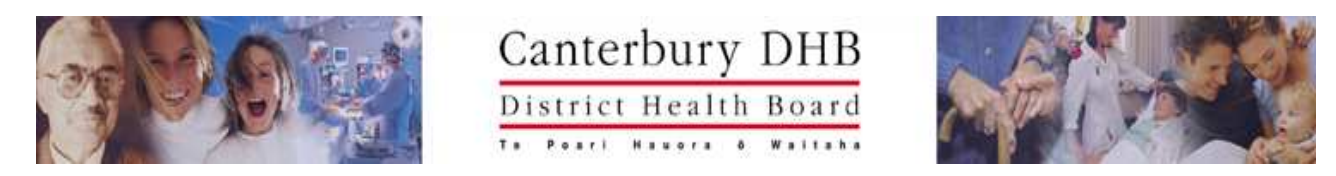


\section{Conclusions and summary}

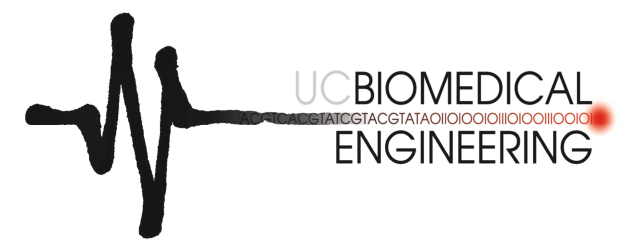

- Minimal cardiac model $\rightarrow$ simulate time varying disease states

- Accurately captures physiological trends and magnitudes

- Accurately captures a wide range of dynamics

- Very Fast simulation methods available

- Integral-based parameter ID $\rightarrow$ patient specific models

- Simulation: ID errors from $0-10 \%$, with $10 \%$ noise

- Animal models: Pulmonary embolism, septic shock (with and without hemofiltration)

Pressures (Total Error) $<8 \%$

Volumes (Total Error) $<5 \%$

- PEEP Therapy prediction: within $10 \%$ error

- Identifiable using a minimal number of common measurements

- Rapid ID method, easily implemented in Matlab

- $\quad$ Rapid ID = Rapid diagnostic feedback

- $\quad$ Future Work = septic shock, ischemia, human trials and other disease states (2008-)
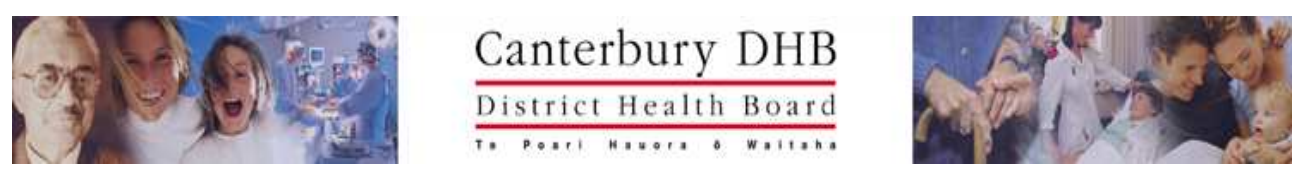


\section{Acknowledgements}

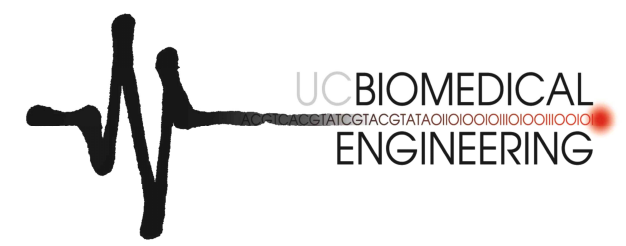

Engineers and Docs

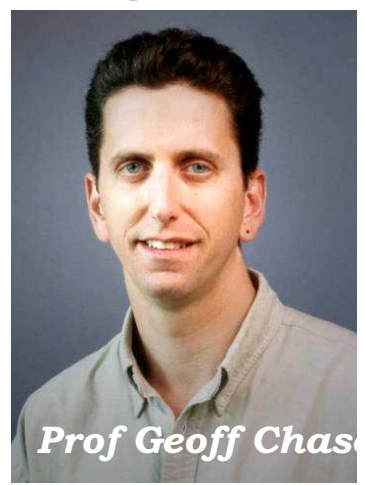

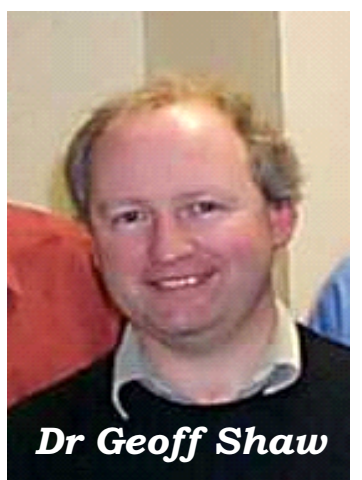

Belgium

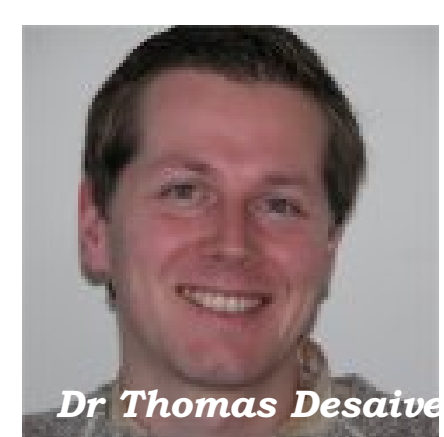

Dr Philippe Kolh

Clinicians:

Dr Alexander Ghuysen

Dr Bernard Lambermont

\section{Honorary Kiwis}

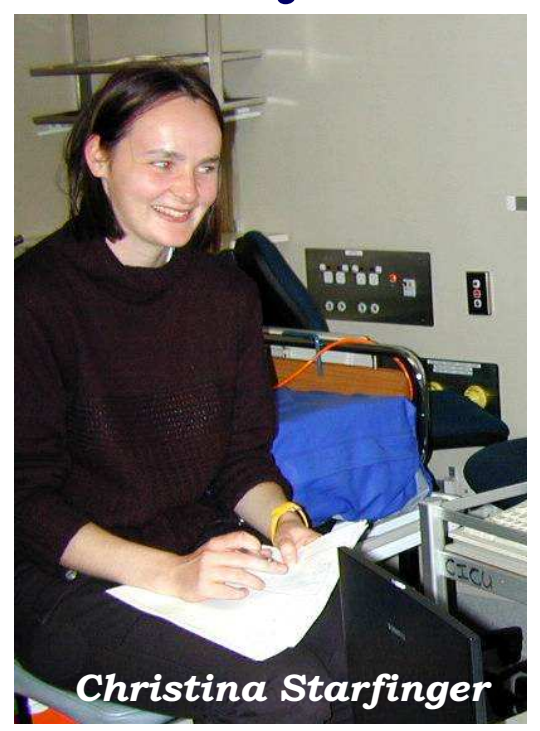

The Danes

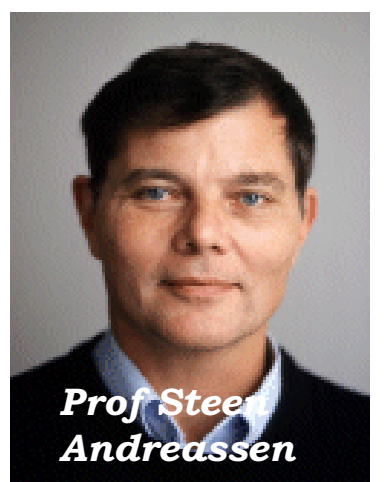

PhD student: French student (6 months):

Katherine Kok Claire Froissart

Summer student (3 months): $\quad$ Research Assistant ( 9 months): James Revie David Stevenson

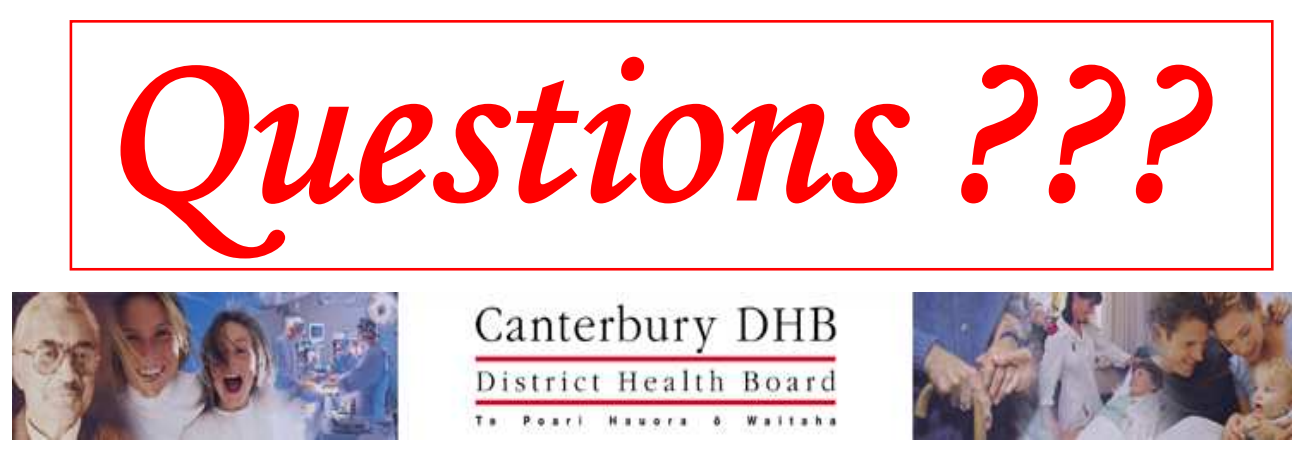

